\title{
Flip-and-forward achieves the optimal diversity-multiplexing tradeoff for the two-hop MIMO relay channel, with two relay antennas
}

\author{
Ramtin Pedarsani, Olivier Lévêque \\ Faculté Informatique et Communications \\ Ecole Polytechnique Fédérale de Lausanne \\ 1015 Lausanne, Switzerland \\ \{ramtin.pedarsani, olivier.leveque\}@epfl.ch
}

\author{
Sheng Yang \\ Département de Télécommunications \\ SUPÉLEC \\ 91192 Gif-sur-Yvette, France \\ sheng.yang@supelec.fr
}

\begin{abstract}
The flip-and-forward scheme introduced in (YangBelfiore, 2007) for handling communication in multi-hop MIMO relay networks was shown to achieve a better diversitymultiplexing tradeoff than pure amplify-and-forward schemes. In the present paper, we show that the flip-and-forward scheme actually achieves the optimal diversity-multiplexing tradeoff for a two-hop relay network, when the relay is equipped with two antennas.
\end{abstract}

\section{INTRODUCTION}

The gain of using multiple antennas for setting up communication over a wireless medium has been widely acknowledged in the literature, starting with the seminal works [1], [2]. For point-to-point channels, the performance of multiple antenna systems is quite well understood by now; in particular, the optimal tradeoff between reliability and rate (also known as diversity-multiplexing tradeoff or DMT) of such systems at high SNR was analyzed in detail in [3].

In wireless networking situations, less is known in general about the communication strategies that allow to reach the optimal performance in terms of both reliability and rate. A scenario of particular interest for wireless networks is the situation where a source wants to communicate to a destination with the help of intermediate relays. A vast literature is available on the subject, which proposes numerous methods to handle communication efficiently (see, e.g., [5], [6], [7], [8], [9], [10], [11], [12], [15], [13], [14] and the references therein). It is worth mentioning that in [14] the authors propose a non-linear scheme, called quantize-mapand-forward, based on random coding arguments, and show that the information theoretic cutset bound is achievable within a constant number of bits. In the present paper, we focus on the scenario where all the nodes are equipped with multiple antenna devices and the source and the destination are too far apart to establish a direct link. The channel between the source and the destination is called a multi-hop MIMO relay channel in this case. Moreover, we consider linear relaying schemes for the sake of simplicity of practical implementation.

A natural and simple scheme to implement for such systems is the amplify-and-forward scheme, which consists in forwarding directly (scaled versions of) the received signals from one relay to the next. At fixed SNR, this scheme is known to suffer from noise amplification at each level, but in the high SNR regime, this issue disappears, provided that the number of relays is fixed and that the SNR is high enough. It was nevertheless observed in [15] that even in this situation, amplify-and-forward schemes do not achieve the optimal diversity gain. The reason is that an additional outage event, referred to as "mismatch" of adjacent channels, is incurred by the multiplication of the channel matrices.

A new strategy was then proposed in [15] to overcome this limitation, which works as follows. A single transmission block is divided into multiple slots. In each slot, the relay antennas forward the received signals with or without flipping the sign, according to a fixed pattern. This strategy, called flip-and-forward, creates an artificial time varying channel within a single slow fading transmission block and recovers the lost diversity caused by the channel mismatch. A lower bound on the DMT of this scheme has been established in [15]. Furthermore, it has been shown that the optimal diversity gain can be achieved, although the DMT optimality of this scheme is still open.

A generalized version of the flip-and-forward scheme, called "rotate-and-forward", was proposed next in [16]. Instead of flipping the sign of the received signal, the relays perform scalar complex rotations in a distributed manner, according to a fixed pattern. It has been shown that when the number of slots goes to infinity, the optimal DMT can be achieved in the case of two relay antennas. However, it may be of less practical interest if the transmission is subject to a stringent delay constraint.

Therefore, whether the simple flip-and-forward scheme with finite number of slots is DMT optimal or not remains a question of interest from both the theoretical and practical point of view. We answer this question in the affirmative in this paper, in the case where the relay has two antennas.

\section{SYSTEM MODEL}

We consider a relay channel with $n$ antennas at the source, $m$ antennas at the destination and $p$ antennas at the relay ${ }^{1}$. We assume no direct link between the source and the destination, so the channel is a two-hop relay channel, and we also assume that the relay is full-duplex. Let $H_{1}$ denote

\footnotetext{
${ }^{1}$ Since we do not consider joint processing of different antennas, the results apply to an arbitrary number of relays and $p$ generally refers to the total number of antennas within the layer of relays.
} 


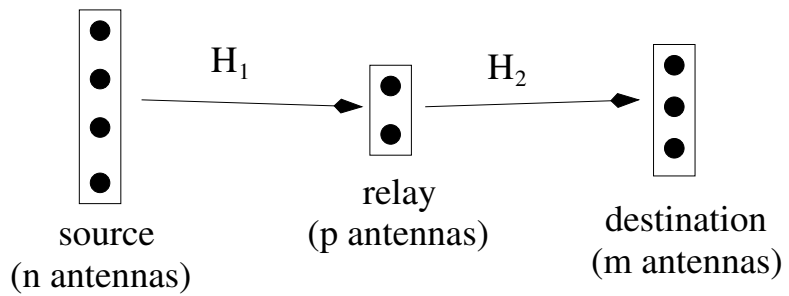

Fig. 1. Two-hop MIMO relay channel.

the $p \times n$ channel matrix between the source and the relay, and $H_{2}$ denote the $m \times p$ channel matrix between the relay and the destination (see Figure 1).

The channel is assumed to be slow-fading, so the channel matrices $H_{1} \in \mathbb{C}^{p \times n}$ and $H_{2} \in \mathbb{C}^{m \times p}$ are fixed during each transmission block. The channel state is known at the receivers, but not at the transmitters; furthermore, the channel matrix entries are assumed to be i.i.d. circularly symmetric complex Gaussian with unit variance.

In the flip-and-forward scheme proposed in [15], the relay flips at each time slot a given number of components of its received signal and then forwards the flipped signal to the destination, according to a fixed pattern. This corresponds to multiplying the received signal vector by a diagonal matrix $\Delta \in \mathcal{F}$ with $\Delta_{i i} \in\{+1,-1\}$ and $\mathcal{F}$ being a set known to all terminals. The channel between the source and the destination at time slot $t$ can therefore be written as

$$
y_{t}=H_{2} \Delta_{t} H_{1} x_{t}+z_{t}, \quad t=1, \ldots, \mathcal{F}
$$

where $x_{t} \in \mathbb{C}^{n}$ and $y_{t} \in \mathbb{C}^{m}$ are the transmitted and received signals, and $z_{t} \in \mathbb{C}^{m}$ is the complex Gaussian noise with covariance matrix $K_{z_{t}}=I_{m}+H_{2} \Delta_{t} \Delta_{t}^{*} H_{2}^{*}=I_{m}+H_{2} H_{2}^{*}$ (where $A^{*}$ denotes the complex-conjugate transpose of $A$ ). Besides, there are $|\mathcal{F}|$ possible matrices $\Delta$; these are chosen sequentially by the relay over $|\mathcal{F}|$ consecutive time slots. Let us denote this chosen sequence by $\Delta_{1}, \ldots, \Delta_{|\mathcal{F}|}$.

For a given realization of the channel matrices $H_{1}$ and $H_{2}$ and i.i.d. signaling at the source (which is shown to be asymptotically optimal in [3]), the average mutual information per channel use between the source and the destination is therefore given by

$$
I(\mathrm{SNR})=\frac{1}{|\mathcal{F}|} \sum_{t=1}^{|\mathcal{F}|} \log \operatorname{det}\left(I+\operatorname{SNR} \Delta_{t} P \Delta_{t} Q\right),
$$

where $P=H_{1} H_{1}^{*}$ and $Q=H_{2}^{*} H_{2}$ are both $p \times p$ matrices.

Correspondingly, the outage probability for a target rate $R=r \log \mathrm{SNR}$ is given by

$$
\mathbb{P}_{\text {out }}(r \log \mathrm{SNR})=\mathbb{P}(I(\mathrm{SNR})<r \log \mathrm{SNR})
$$

and the scheme is said to achieve simultaneously multiplexing gain $r$ and diversity gain $d$ if

$$
\mathbb{P}_{\text {out }}(r \log \mathrm{SNR}) \doteq \mathrm{SNR}^{-d},
$$

where $f(\mathrm{SNR}) \doteq g(\mathrm{SNR})$ means

$$
\lim _{\mathrm{SNR} \rightarrow \infty} \frac{\log (f(\mathrm{SNR}))}{\log \mathrm{SNR}}=\lim _{\mathrm{SNR} \rightarrow \infty} \frac{\log (g(\mathrm{SNR}))}{\log \mathrm{SNR}} .
$$

The diversity-multiplexing tradeoff is therefore given by the curve $d(r)$ defined as

$$
d(r)=\lim _{\mathrm{SNR} \rightarrow \infty}-\frac{\log \left(\mathbb{P}_{\text {out }}(r \log \mathrm{SNR})\right)}{\log \mathrm{SNR}},
$$

The DMT curve $d(r)$ established by the outage formulation can be achieved with approximately universal codes. More specifically, the equivalent channel of the flip-and-forward scheme is a parallel $n \times m$ MIMO channel. Therefore, the universal codes for such channels (e.g., the perfect codes proposed in [4]) can be used to attain the optimal DMT.

\section{DMT ANALYSIS}

Using a simple cutset bound argument, it can be shown that the DMT curve of the two-hop relay channel is upper bounded by that of a classical $(n \wedge m) \times p$ MIMO channel, where $n \wedge m=\min (n, m)$.

In this section, we show that the DMT achieved by the flipand-forward scheme is optimal when the number of antennas at the relay is equal to $p=2$. This result therefore confirms that of [16] obtained for the rotate-and-forward scheme under the same assumptions on the number of antennas at the relay. The proof follows nevertheless a quite divergent path here. In particular and surprisingly perhaps, the case where the number of antennas at both the source and the destination $n=m=2$ exhibits some subtle differences in the analysis with all the other cases $(n>2$ and $m>2)$.

Theorem 1: When the number of antennas at the relay is equal to $p=2$, the DMT achieved by the flip-and-forward scheme is that of an $(n \wedge m) \times 2$ classical MIMO channel, i.e.

$$
d(r)= \begin{cases}2(n \wedge m)-(n \wedge m)+1) r, & \text { if } r \in[0,1], \\ ((n \wedge m)-1)(2-r), & \text { if } r \in[1,2] .\end{cases}
$$

Proof: The strategy for the proof is as follows. We first obtain a lower bound $I_{\mathrm{LB}}(\mathrm{SNR})$ on the mutual information (1), which can be written in terms of a set of independent random variables, whose distributions are known. This lower bound gives an upper bound on the outage probability

$$
\begin{aligned}
\mathbb{P}_{\text {out }}(r \log \mathrm{SNR}) & =\mathbb{P}(I(\mathrm{SNR})<r \log \mathrm{SNR}) \\
& \leq \mathbb{P}\left(I_{\mathrm{LB}}(\mathrm{SNR})<r \log \mathrm{SNR}\right)
\end{aligned}
$$

which in turn yields a lower bound on the diversity order $d(r) \geq d_{\mathrm{LB}}(r)$. From the joint distribution of the random variables involved in $I_{\mathrm{LB}}(\mathrm{SNR})$, we compute the diversity order $d_{\mathrm{LB}}(r)$, following the methodology of [3], and show that this lower bound matches the expression (2).

In the case with $p=2$, the flip set $\mathcal{F}$ has two members: $I$ and $\operatorname{diag}(1,-1)$. The expression (1) for the mutual information may therefore be rewritten as

$I(\mathrm{SNR})=\frac{1}{2} \log \operatorname{det}(I+\mathrm{SNR} P Q)+\frac{1}{2} \log \operatorname{det}(I+\mathrm{SNR} \widetilde{P} Q)$

where

$$
\widetilde{P}=\left(\begin{array}{cc}
1 & 0 \\
0 & -1
\end{array}\right) P\left(\begin{array}{cc}
1 & 0 \\
0 & -1
\end{array}\right) .
$$


Using the fact that for a $2 \times 2$ matrix $A$,

$$
\operatorname{det}(I+A)=1+a_{11}+a_{22}+\operatorname{det}(A),
$$

we obtain, after some manipulations,

$$
\begin{aligned}
& I(\mathrm{SNR})=\frac{1}{2} \log \left(\left(1+\mathrm{SNR}\left(p_{11} q_{11}+p_{22} q_{22}\right)\right.\right. \\
& \left.\left.+\mathrm{SNR}^{2} \operatorname{det}(P) \operatorname{det}(Q)\right)^{2}-\mathrm{SNR}^{2}\left(p_{12} q_{21}+p_{21} q_{12}\right)^{2}\right)
\end{aligned}
$$

Notice that $p_{11}=\left\|h_{1}^{(1)}\right\|^{2}$ and $p_{22}=\left\|h_{2}^{(1)}\right\|^{2}$, where $h_{1}^{(1)}$ and $h_{2}^{(1)}$ denote the first and second rows of $H_{1}$ respectively (of length $n$ each). The random variables $A_{1}=p_{11}$, $A_{2}=p_{22}$ are therefore i.i.d. with common pdf $p_{A}(a)=$ $a^{n-1} e^{-a} / \Gamma(n)$. Likewise, $q_{11}=\left\|h_{1}^{(2)}\right\|^{2}$ and $q_{22}=\left\|h_{2}^{(2)}\right\|^{2}$, where $h_{1}^{(2)}$ and $h_{2}^{(2)}$ denote this time the first and second columns of $H_{2}$ respectively (of length $m$ each). The random variables $B_{1}=q_{11}$ and $B_{2}=q_{22}$ are therefore i.i.d. with common pdf $p_{B}(b)=b^{m-1} e^{-b} / \Gamma(m)$. Notice also that the four random variables $A_{1}, A_{2}, B_{1}$ and $B_{2}$ are independent. Furthermore, defining

$$
w=\frac{p_{12}}{\sqrt{p_{11} p_{22}}} \quad \text { and } \quad z=\frac{q_{12}}{\sqrt{q_{11} q_{22}}}
$$

and noticing that $p_{12}=\overline{p_{21}}$ and $q_{12}=\overline{q_{21}}$, we obtain

$$
\begin{aligned}
\left(p_{12} q_{21}+p_{21} q_{12}\right)^{2} & =4\left(\operatorname{Re}\left(p_{12} \overline{q_{12}}\right)\right)^{2} \\
& =4 p_{11} p_{22} q_{11} q_{22}(\operatorname{Re}(w \bar{z}))^{2} .
\end{aligned}
$$

In turn, writing $w=|w| e^{i \phi}$ and $z=|z| e^{i \theta}$, the last term on the right-hand side may be rewritten as

$$
(\operatorname{Re}(w \bar{z}))^{2}=|w|^{2}|z|^{2} \cos ^{2}(\phi-\theta) .
$$

Let us define the following three random variables:

$U_{1}=1-|w|^{2}, \quad U_{2}=1-|z|^{2} \quad$ and $\quad V=1-\cos ^{2}(\phi-\theta)$.

In the appendix, it is shown that these three random variables are mutually independent, as well as independent from $A_{1}, A_{2}, B_{1}, B_{2}$. Moreover, their pdfs are given by

$$
\begin{aligned}
p_{U_{1}}\left(u_{1}\right) & =(n-1) u_{1}^{n-2} 1_{\left\{0 \leq u_{1} \leq 1\right\}}, \\
p_{U_{2}}\left(u_{2}\right) & =(m-1) u_{2}^{m-2} 1_{\left\{0 \leq u_{2} \leq 1\right\}}, \\
p_{V}(v) & =\frac{1}{\pi \sqrt{v(1-v)}} 1_{\{0 \leq v \leq 1\}} .
\end{aligned}
$$

Furthermore, observe that

$$
\begin{aligned}
& \operatorname{det}(P)=p_{11} p_{22}-\left|p_{12}\right|^{2}=A_{1} A_{2} U_{1}, \\
& \operatorname{det}(Q)=q_{11} q_{22}-\left|q_{12}\right|^{2}=B_{1} B_{2} U_{2} .
\end{aligned}
$$

So the expression (3) may finally be rewritten as

$$
\begin{aligned}
I(\mathrm{SNR}) & = \\
\frac{1}{2} \log ((1 & \left.+\operatorname{SNR}\left(A_{1} B_{1}+A_{2} B_{2}\right)+\mathrm{SNR}^{2} A_{1} A_{2} B_{1} B_{2} U_{1} U_{2}\right)^{2} \\
& \left.-4 \mathrm{SNR}^{2} A_{1} A_{2} B_{1} B_{2}\left(1-U_{1}\right)\left(1-U_{2}\right)(1-V)\right)
\end{aligned}
$$

Although this expression for the mutual information only contains independent random variables, it also contains a minus sign that prevents us from deducing easily the corresponding DMT curve. Instead, we compute a lower bound on the DMT curve from the following lower bound on the mutual information.

Lemma 1: The mutual information is lower bounded by

$$
\begin{aligned}
I(\mathrm{SNR}) \geq & I_{\mathrm{LB}}(\mathrm{SNR})= \\
\frac{1}{2} \log (1+ & 2 \mathrm{SNR}\left(A_{1} B_{1}+A_{2} B_{2}\right)+2 \mathrm{SNR}^{2} A_{1} A_{2} B_{1} B_{2} V \\
& +2 \mathrm{SNR}^{3} A_{1} A_{2} B_{1} B_{2}\left(A_{1} B_{1}+A_{2} B_{2}\right) U_{1} U_{2} \\
& \left.+\mathrm{SNR}^{4}\left(A_{1} A_{2} B_{1} B_{2} U_{1} U_{2}\right)^{2}\right)
\end{aligned}
$$

The proof of this lemma is given in the appendix.

We are now in position to compute the DMT curve corresponding to this lower bound. The random variables in (4) are independent and their joint pdf is given by

$$
\begin{aligned}
& p_{A_{1}, A_{2}, B_{1}, B_{2}, U_{1}, U_{2}, V}\left(a_{1}, a_{2}, b_{1}, b_{2}, u_{1}, u_{2}, v\right) \\
& =C_{n, m} a_{1}^{n-1} e^{-a_{1}} a_{2}^{n-1} e^{-a_{2}} b_{1}^{m-1} e^{-b_{1}} b_{2}^{m-1} e^{-b_{2}} \\
& \quad \times u_{1}^{n-2} 1_{\left\{0 \leq u_{1} \leq 1\right\}} u_{2}^{m-2} 1_{\left\{0 \leq u_{2} \leq 1\right\}} \frac{1}{\sqrt{v(1-v)}} 1_{\{0 \leq v \leq 1\}}
\end{aligned}
$$

Following [3], let us then operate the following change of variables:

$$
\begin{aligned}
& A_{1}=\mathrm{SNR}^{-\alpha_{1}}, \quad A_{2}=\mathrm{SNR}^{-\alpha_{2}}, \quad B_{1}=\mathrm{SNR}^{-\beta_{1}}, \\
& B_{2}=\mathrm{SNR}^{-\beta_{2}}, \quad U_{1}=\mathrm{SNR}^{-\gamma_{1}}, \quad U_{2}=\mathrm{SNR}^{-\gamma_{2}}, \\
& V=\mathrm{SNR}^{-\delta} \text {. }
\end{aligned}
$$

The joint pdf of the new variables becomes

$$
\begin{aligned}
p( & \left.\alpha_{1}, \alpha_{2}, \beta_{1}, \beta_{2}, \gamma_{1}, \gamma_{2}, \delta\right)=C_{n, m}(\log (\mathrm{SNR}))^{7} \\
& \times \mathrm{SNR}^{-\left(n\left(\alpha_{1}+\alpha_{2}\right)+m\left(\beta_{1}+\beta_{2}\right)+(n-1) \gamma_{1}+(m-1) \gamma_{2}+\delta / 2\right)} \\
& \times e^{-\left(\mathrm{SNR}^{-\alpha_{1}}+\mathrm{SNR}^{-\alpha_{2}}+\mathrm{SNR}^{-\beta_{1}}+\mathrm{SNR}^{-\beta_{2}}\right)} \\
& \times 1_{\left\{\gamma_{1} \geq 0, \gamma_{2} \geq 0, \delta \geq 0\right\}} \frac{1}{\sqrt{1-\mathrm{SNR}^{-\delta}}} .
\end{aligned}
$$

The upper bound on the outage probability therefore reads

$$
\begin{aligned}
& \mathbb{P}_{\text {out }}(r \log \mathrm{SNR}) \leq \mathbb{P}\left(I_{\mathrm{LB}}(\mathrm{SNR})<r \log \mathrm{SNR}\right) \\
& =\int_{D_{r}} d \alpha_{1} d \alpha_{2} d \beta_{1} d \beta_{2} d \gamma_{1} d \gamma_{2} d \delta p\left(\alpha_{1}, \alpha_{2}, \beta_{1}, \beta_{2}, \gamma_{1}, \gamma_{2}, \delta\right)
\end{aligned}
$$

where

$D_{r}=\left\{\alpha_{1}, \alpha_{2}, \beta_{1}, \beta_{2}, \gamma_{1}, \gamma_{2}, \delta \in \mathbb{R}: I_{\mathrm{LB}}(\mathrm{SNR})<r \log \mathrm{SNR}\right\}$

This expression can be further approximated asymptotically by

$$
\begin{aligned}
& \mathbb{P}\left(I_{\mathrm{LB}}(\mathrm{SNR})<r \log \mathrm{SNR}\right) \\
& \doteq \int_{\widetilde{D}_{r}} d \alpha_{1} d \alpha_{2} d \beta_{1} d \beta_{2} d \gamma_{1} d \gamma_{2} d \delta \\
& \quad \times \mathrm{SNR}^{-\left(n\left(\alpha_{1}+\alpha_{2}\right)+m\left(\beta_{1}+\beta_{2}\right)+(n-1) \gamma_{1}+(m-1) \gamma_{2}+\delta / 2\right)} .
\end{aligned}
$$

where

$\widetilde{D}_{r}=\left\{\alpha_{1}, \alpha_{2}, \beta_{1}, \beta_{2}, \gamma_{1}, \gamma_{2}, \delta \geq 0: I_{\mathrm{LB}}(\mathrm{SNR})<r \log \mathrm{SNR}\right\}$ 
Using Laplace's integration's method, we deduce that

$d_{\mathrm{LB}}(r)=$

$\min \left\{n\left(\alpha_{1}+\alpha_{2}\right)+m\left(\beta_{1}+\beta_{2}\right)+(n-1) \gamma_{1}+(m-1) \gamma_{2}+\frac{\delta}{2}\right\}$

subject to $\alpha_{1}, \alpha_{2}, \beta_{1}, \beta_{2}, \gamma_{1}, \gamma_{2}, \delta \geq 0$ and

$\max \left(0,1-\alpha_{1}-\beta_{1}, 1-\alpha_{2}-\beta_{2}, 2-\alpha_{1}-\beta_{1}-\alpha_{2}-\beta_{2}-\delta\right.$,

$3-\alpha_{1}-\beta_{1}-\alpha_{2}-\beta_{2}-\min \left(\alpha_{1}+\beta_{1}, \alpha_{2}+\beta_{2}\right)-\gamma_{1}-\gamma_{2}$,

$4-2\left(\alpha_{1}-\beta_{1}-\alpha_{2}-\beta_{2}-\gamma_{1}-\gamma_{2}\right)<r$.

The solution of this optimization problem depends now on the values of $m$ and $n$.

$$
\begin{aligned}
& \text { Case } m=n=2 \text {. For } 0 \leq r \leq 0.5 \text {, we have } \\
& \left.\begin{array}{l}
\alpha_{1}=\alpha_{2}=\beta_{1}=\beta_{2}=0.5-r \\
\gamma_{1}=\gamma_{2}=2 r \\
\delta=2 r
\end{array}\right\} \Rightarrow d_{\mathrm{LB}}(r)=4-3 r .
\end{aligned}
$$

For $0.5 \leq r \leq 1$, we have

$$
\left.\begin{array}{l}
\alpha_{1}=\alpha_{2}=\beta_{1}=\beta_{2}=0 \\
\gamma_{1}=\gamma_{2}=1.5-r \\
\delta=2-2 r
\end{array}\right\} \Rightarrow d_{\mathrm{LB}}(r)=4-3 r .
$$

For $1 \leq r \leq 2$, we have

$$
\left.\begin{array}{l}
\alpha_{1}=\alpha_{2}=\beta_{1}=\beta_{2}=0 \\
\gamma_{1}=\gamma_{2}=1-0.5 r \\
\delta=0
\end{array}\right\} \Rightarrow d_{\mathrm{LB}}(r)=2-2 r .
$$

These three expressions match the expression (2) for the considered intervals, so the proof of Theorem 1 is complete for $m=n=2$.

Case $m>2$ or $n>2$. Without loss of generality, we assume that $n \geq m$. For $0 \leq r \leq 1$ we have

$$
\left.\begin{array}{l}
\alpha_{1}=\alpha_{2}=\gamma_{1}=\delta=0 \\
\beta_{1}=\beta_{2}=1-r \\
\gamma_{2}=r
\end{array}\right\} \Rightarrow d_{\mathrm{LB}}(r)=2 m-(m+1) r .
$$

For $1 \leq r \leq 2$ we have

$$
\left.\begin{array}{l}
\alpha_{1}=\alpha_{2}=\beta_{1}=\beta_{2}=0 \\
\gamma_{1}=\delta=0 \\
\gamma_{2}=2-r
\end{array}\right\} \Rightarrow d_{\mathrm{LB}}(r)=(m-1)(2-r) .
$$

These two expressions match the expression (2) for the intervals considered, so the proof of Theorem 1 is complete for arbitrary values of $m$ and $n$.

It is remarkable that for a target multiplexing gain $r$ ranging between 0 and 1, the typical outage event in the case $m=n=2$ is of a different nature than in the other cases.

In the first case ( $m=n=2)$, the main outage event at $r=0.5$, corresponding to $\gamma_{1}=\gamma_{2}=\delta=1$, is caused by the two matrices $H_{1}$ and $H_{2}$ being rank 1, i.e. $h_{2}^{(1)}=c_{1} h_{1}^{(1)}$ and $h_{2}^{(2)}=c_{2} h_{1}^{(2)}$, as well as the alignment in the complex plane of the two proportionality factors $c_{1}$ and $c_{2}$.

In all the other cases (assuming without loss of generality that $n \geq m$ ), the main outage event at $r=0.5$ corresponds to $\beta_{1}=\beta_{2}=\gamma_{2}=0.5$ and is therefore mainly determined by the matrix $\mathrm{H}_{2}$ having relatively small entries (notice that in the case where $n=m>2$, other outage events give rise to the same diversity, but they are not of the same nature as in the $n=m=2$ case).

\section{Conclusion And Perspectives}

In this paper, we have proved that the flip-and-forward scheme presented in [15] is DMT optimal when the relay has two antennas. This shows that the diversity-multiplexing tradeoff for a two-hop relay channel can be achieved with a practical linear relaying scheme. We actually believe that the optimality of the flip-and-forward scheme holds in more generality, i.e. with either a larger number of antennas in the relay or a larger number of relays. However, the proof of this fact remains open.

\section{APPENDIX}

Joint distribution of the random variables $U_{1}, U_{2}$ and $V$. Let us first consider the random variable $w=\frac{p_{12}}{\sqrt{p_{11} p_{22}}}$. Denoting by $h_{1}^{(1)}$ and $h_{2}^{(1)}$ the first and second rows of $H_{1}$ respectively, we obtain

$$
w=\frac{h_{1}^{(1)} \cdot\left(h_{2}^{(1)}\right)^{*}}{\left\|h_{1}^{(1)}\right\|\left\|h_{2}^{(1)}\right\|} .
$$

Since the row vectors $h_{1}^{(1)}$ and $h_{2}^{(1)}$ have i.i.d. complex Gaussian entries, the distribution of each of them is unitarily invariant. So $\frac{h_{1}^{(1)}}{\left\|h_{1}^{(1)}\right\|}$ may be expressed as

$$
\frac{h_{1}^{(1)}}{\left\|h_{1}^{(1)}\right\|}=[1,0,0, \cdots, 0] U
$$

where $U \in \mathbb{C}^{n \times n}$ is uniformly distributed on the set of $n \times n$ unitary matices (Haar distribution). Therefore,

$$
w=[1,0,0, \cdots, 0] U \frac{\left(h_{2}^{(1)}\right)^{*}}{\left\|h_{2}^{(1)}\right\|},
$$

which has the same distribution as

$$
[1,0,0, \cdots, 0] \cdot \frac{\left(h_{2}^{(1)}\right)^{*}}{\left\|h_{2}^{(1)}\right\|}=\frac{\overline{h_{21}^{(1)}}}{\left\|h_{2}^{(1)}\right\|}
$$

i.e.

$$
|w|^{2} \sim \frac{\left|h_{21}^{(1)}\right|^{2}}{\left\|h_{2}^{(1)}\right\|^{2}}
$$

So the random variable $U_{1}=1-|w|^{2}$ is distributed as

$$
U_{1} \sim \frac{\sum_{k=2}^{n}\left|h_{2 k}^{(1)}\right|^{2}}{\left\|h_{2}^{(1)}\right\|^{2}}
$$

Lemma 2: If $X$ and $Y$ are two independent Gammadistributed random variables with parameters $\left(k_{1}, \theta\right)$ and $\left(k_{2}, \theta\right)$ respectively, then the random variable $Z=\frac{X}{X+Y}$ 
is independent of $X$ and $Y$ and is distributed according to the Beta distribution with parameters $\left(k_{1}, k_{2}\right)$, i.e.

$$
\begin{aligned}
p_{X}(x) & =x^{k_{1}-1} \frac{e^{-x / \theta}}{\theta^{k_{1}} \Gamma\left(k_{1}\right)} 1_{\{x \geq 0\}}, \\
p_{Y}(y) & =y^{k_{2}-1} \frac{e^{-y / \theta}}{\theta^{k_{2}} \Gamma\left(k_{2}\right)} 1_{\{y \geq 0\}}, \\
p_{Z}(z) & =\frac{z^{k_{1}-1}(1-z)^{k_{2}-1}}{\int_{0}^{1} u^{k_{1}-1}(1-u)^{k_{2}-1} d u} .
\end{aligned}
$$

Proof. See [17].

Note that the random variables $\left|h_{2 k}^{(1)}\right|^{2}$ are i.i.d. exponential random variables. Therefore,

$$
\begin{aligned}
& X=\sum_{k=2}^{n}\left|h_{2 k}^{(1)}\right|^{2} \sim \operatorname{Gamma}(n-1,1), \\
& Y=\left|h_{21}^{(1)}\right|^{2} \sim \operatorname{Gamma}(1,1) .
\end{aligned}
$$

Hence, due to Lemma 2,

$$
U_{1} \sim \frac{X}{X+Y} \sim \operatorname{Beta}(n-1,1)
$$

which is the order statistics of the largest point among $n-1$ i.i.d. points uniformly distributed on the interval $[0,1]$, i.e.

$$
p_{U_{1}}\left(u_{1}\right)=(n-1) u_{1}^{n-2} 1_{\left\{0 \leq u_{1} \leq 1\right\}}
$$

Similarly, it can be shown that

$$
p_{U_{2}}\left(u_{2}\right)=(m-1) u_{2}^{m-2} 1_{\left\{0 \leq u_{2} \leq 1\right\}}
$$

and $U_{1}$ and $U_{2}$ are clearly independent, as $H_{1}$ and $H_{2}$ are.

Furthermore, for any fixed argument $\alpha \in[0,2 \pi], w e^{i \alpha} \sim$ $w$. So, the random variable $\phi$, which is the argument of $w$, is uniformly distributed on $[0,2 \pi]$, and is independent of all the random variables $\left|h_{2 k}^{(1)}\right|^{2}$ since the phase and amplitude of a circularly symmetric Gaussian random variable are independent. The same argument holds for $z=|z| e^{i \theta}$. Therefore, $\phi-\theta$ is also uniformly distributed on $[0,2 \pi]$. We deduce that the random variable $V=1-\cos ^{2}(\phi-\theta)$, which is a function of $\phi$ and $\theta$ is also independent of $U_{1}$ and $U_{2}$, and has pdf

$$
p_{V}(v)=\frac{1}{\pi \sqrt{v(1-v)}} 1_{\{0 \leq v \leq 1\}} .
$$

This completes the proof.

Proof of Lemma 1.

Let us compute

$$
\begin{gathered}
\left(1+\operatorname{SNR}\left(A_{1} B_{1}+A_{2} B_{2}\right)+\mathrm{SNR}^{2} A_{1} A_{2} B_{1} B_{2} U_{1} U_{2}\right)^{2} \\
-4 \mathrm{SNR}^{2} A_{1} A_{2} B_{1} B_{2}\left(1-U_{1}\right)\left(1-U_{2}\right)(1-V) \\
=1+2 \mathrm{SNR}\left(A_{1} B_{1}+A_{2} B_{2}\right)+\operatorname{SNR}^{4}\left(A_{1} A_{2} B_{1} B_{2} U_{1} U_{2}\right)^{2} \\
+2 \mathrm{SNR}^{3} A_{1} A_{2} B_{1} B_{2}\left(A_{1} B_{1}+A_{2} B_{2}\right) U_{1} U_{2} \\
+\operatorname{SNR}^{2}\left(\left(A_{1} B_{1}+A_{2} B_{2}\right)^{2}+2 A_{1} A_{2} B_{1} B_{2} U_{1} U_{2}\right. \\
\left.\quad-4 A_{1} A_{2} B_{1} B_{2}\left(1-U_{1}\right)\left(1-U_{2}\right)(1-V)\right)
\end{gathered}
$$

The last term in parentheses is given by

$$
\begin{aligned}
& \left(A_{1} B_{1}\right)^{2}+\left(A_{2} B_{2}\right)^{2}+A_{1} A_{2} B_{1} B_{2}\left(2+2 U_{1} U_{2}\right. \\
& \left.-4\left(1-U_{1}-U_{2}-V+U_{1} U_{2}+U_{1} V+U_{2} V-U_{1} U_{2} V\right)\right) \\
& =\left(A_{1} B_{1}-A_{2} B_{2}\right)^{2}+A_{1} A_{2} B_{1} B_{2}\left(-2 U_{1} U_{2}\right. \\
& \left.\quad+4\left(U_{1}+U_{2}+V-U_{1} V-U_{2} V+U_{1} U_{2} V\right)\right) \\
& \geq 2 A_{1} A_{2} B_{1} B_{2} V .
\end{aligned}
$$

Indeed, $U_{1}, U_{2}$ and $V$ belong to $[0,1]$, so

$$
\begin{gathered}
-2 U_{1} U_{2}+4\left(U_{1}+U_{2}+V-U_{1} V-U_{2} V+U_{1} U_{2} V\right) \\
\geq 3\left(U_{1}+U_{2}\right)+2 V-3\left(U_{1}+U_{2}\right)+0 \geq 2 V .
\end{gathered}
$$

This completes the proof.

\section{REFERENCES}

[1] G. J. Foschini and M. J. Gans, "On Limits of Wireless Communications in a Fading Environment when Using Multiple Antennas", Wireless Personal Communications, vol. 6, no. 3, March 1998, pp. 311-335.

[2] E. Telatar, "Capacity of Multi-antenna Gaussian Channels", European Trans. on Telecommunications, vol. 10, no. 6, June 1999, pp. 585-596.

[3] L. Zheng and D. Tse, "Diversity and Multiplexing: A Fundamental Tradeoff in Multiple Antenna Channels", IEEE Trans. Inf. Theory, vol. 49, no. 5, pp. 1073-96, May 2003.

[4] S. Yang, J.-C. Belfiore, and G. Rekaya, "Perfect space-time block codes for parallel MIMO channels", in Proc. IEEE International Symposium on Information Theory 2006, Seattles, Jul. 2006.

[5] J. N. Laneman and G. W. Wornell, "Distributed space-time-coded protocols for exploiting cooperative diversity in wireless networks," IEEE Trans. Inf. Theory, vol. 49, no. 10, pp. 2415-2425, Oct. 2003.

[6] J. N. Laneman, D. N. C. Tse, and G. W. Wornell, "Cooperative diversity in wireless networks: Efficient protocols and outage behavior," IEEE Trans. Inf. Theory, vol. 50, no. 12, pp. 3062-3080, Dec. 2004.

[7] T. Hunter, S. Sanayei, and A. Nosratinia, "Outage analysis of coded cooperation," IEEE Trans. Inf. Theory, vol. 52, no. 2, pp. 375-391, Feb. 2006.

[8] R. U. Nabar, H. Bölcskei, and F. W. Kneubühler, "Fading relay channels: Performance limits and space-time signal design," IEEE J. Sel. Areas Commun., vol. 22, no. 6, pp. 1099-1109, Aug. 2004

[9] K. Azarian, H. El Gamal, and P. Schniter, "On the achievable diversitymultiplexing tradeoff in half-duplex cooperative channels," IEEE Trans. Inf. Theory, vol. 51, no. 12, pp. 4152-4172, Dec. 2005.

[10] S. Yang and J.-C.'Belfiore, "Towards the optimal amplify-and-forward cooperative diversity scheme," IEEE Trans. Inf. Theory, vol. 53, no. 9, pp. 3114-3126, Sep. 2007.

[11] M. Yuksel and E. Erkip, "Multi-antenna cooperative wireless systems: A diversity multiplexing tradeoff perspective," IEEE Trans. Inf. Theory, vol. 53, no. 10, pp. 3371-3393, Oct. 2007.

[12] Y. Jing and B. Hassibi, "Distributed space-time coding in wireless relay networks," IEEE Trans. Wireless Commun., vol. 5, no. 12, pp. 3524-3536, Dec. 2006.

[13] P. Elia, K. Vinodh, M. Anand, and P. V. Kumar, "D-MG tradeoff and optimal codes for a class of af and df cooperative communication protocols," IEEE Trans. Inf. Theory, vol. 55, no. 7, pp. 3161-3185, Jul. 2009.

[14] S. Avestimehr, S. Diggavi, and D. N. C. Tse, "Wireless network information flow: a deterministic approach," IEEE Trans. Inf. Theory, June 2009, submitted. [Online]. Available: http://arxiv.org/ abs/0906.5394

[15] S. Yang, J.-C. Belfiore, "Diversity of MIMO Multihop Relay Channels", Aug. 2007. [Online]. Available: http://arxiv.org/abs/ 0708.0386.

[16] S. Yang, J.-C. Belfiore, "Distributed Rotation Recovers Spatial Diversity", submitted to the IEEE International Symposium on Information Theory 2010, Austin, Texas, June 2010.

[17] R. V. Hogg, A. T. Craig, "Introduction to Mathematical Statistics", Macmillan, New York, 1978. 